\title{
Implementation of Object Relational Data Base Management on Depreciation System Using ORACL
}

Asmaa Yaseen Hammo

asmahammo@uomosul.edu.iq

College of Computer Sciences and

Mathematics, University of Mosul, Iraq

Received on: 22/12/2003
Alaa F. Saeed

General Directorate of North

Electricity Distribution

Ministry of Electricity, Iraq

Accepted on: 22/08/2004

\section{ABSTRACT}

This research implements concept of Object Relational DataBases Management System ORDBMS on depreciation system because it's considered as a complex model. ORDBMS abstracts the level of writing system by adding data types defined by user (user define type UDT) which represents business objects, these objects are stored in database as a column on table or object table which can be accessed by using methods defined by user (user define function UDF). Object reference REFs is used instead of multiple relational between tables. Multiple data are implemented using collection data type (Varray, Nested table) without the need for extra tables. The application is programmed using oracle8 software because it has the propriety of an object relational database.

Keywards: Object Relational DataBases Management System (ORDBMS), ORACL, Varray, Nested Table, user define type (UDT), user define function (UDF).

$$
\begin{aligned}
& \text { تطبيق نظام إدارة قواعد البيانات الكيانية العلاثقية على نظام الاندثارات } \\
& \text { باستخدام لغة أو راكل } \\
& \text { أسماء ياسين حمو } \\
& \text { كلية علوم الحاسبات والرياضيات } \\
& \text { جامعة الموصل } \\
& \text { تاريخ قبول البحث: 2004/08/22 }
\end{aligned}
$$

يعرض البحث كيفية تطبيق مفاهيم نظام إدارة قواعد البيانات الكيانية العلائقية (ORDBMS) نو Object Relational Data Base Management System نموذجاً معقداً عن (ORDBMS) إذ يقوم برفع مستوى التجريد الذي يكتب فيه النظام من خلال إضـافة أنواع بيانات عرفها المستخدم User Define Type(UDT) وهي تمثل كيانات إدارة الأعمال، وهذه الكيانات تخزن في قاعدة البيانات كعمود في الجدول أو كجدول كيانات، ويتم

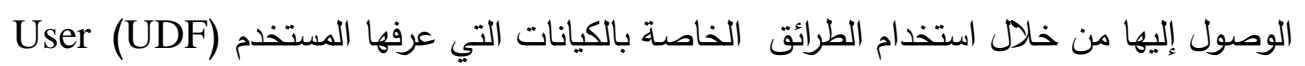


References (REFs) وكان لاستخدام مراجع الكيان Define Function

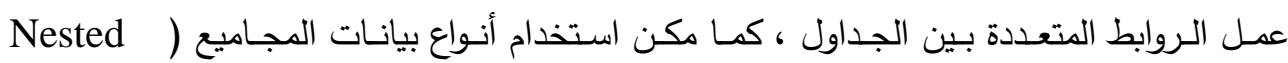
(table,Varray

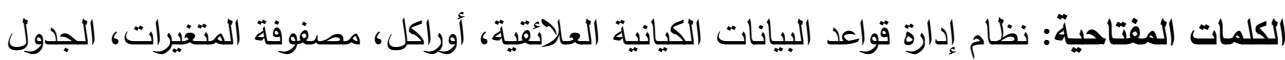
المتداخل، بيانات عرفها المستخدم، طرائق عرفها المستخدم. 1- تاريخ نظم قواعد البيانات

ظهر أول نظام خاص بإدارة قواعد البيانات في أواخر عام 1960 وتطور ليعرف بنظام الملفاتFile Systems الذي يقوم بخزن كميات كبيرة من البيانات ولفترات طويلة من الزمن ولكنه

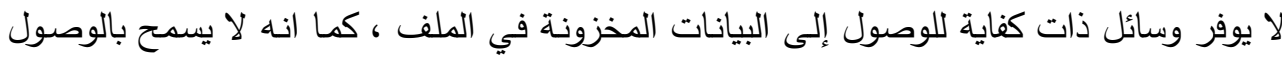
المتزامن لأكثر من مستخدم إلى الملف نفسه [15] ـ أعقب ذلك تطور نظام الملفات إلى النموذج لئه

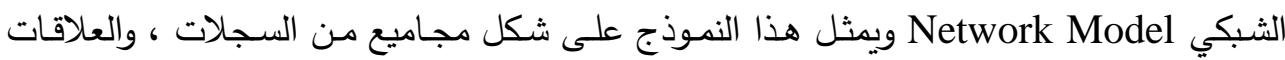

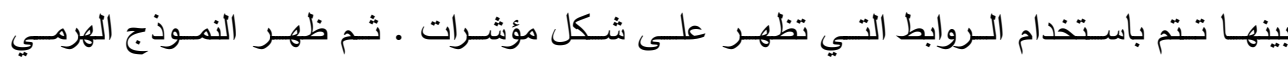

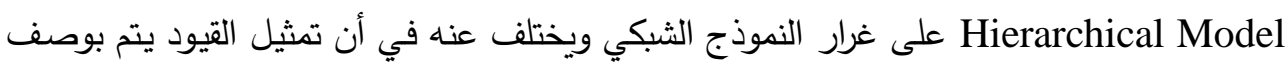

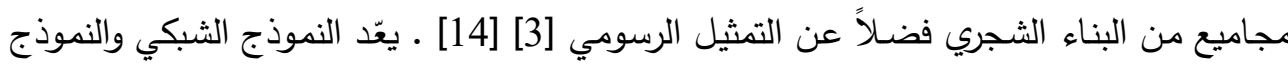

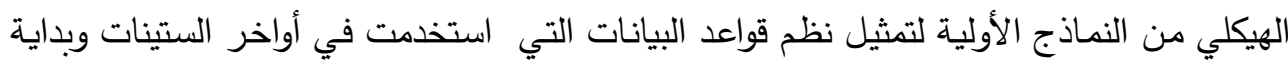

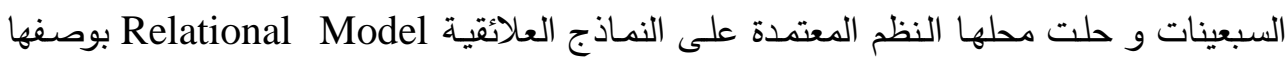
منافساً كبيراً [15] ويقوم هذا النموذج بتمثيل البيانات والعلاقات بينها من خلال الجداول التي تحتوي

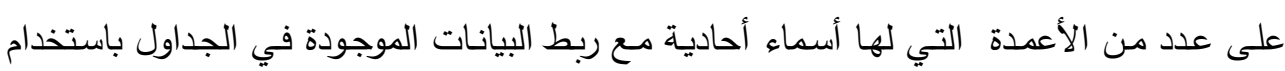

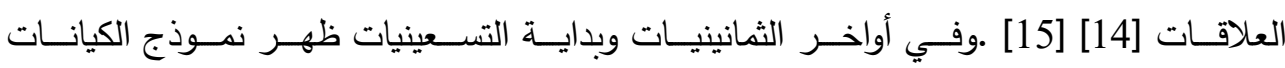
الموجهةObject Oriented Model لأسباب كثيرة منها فقدان الهيكلية في الجداول العلائقية

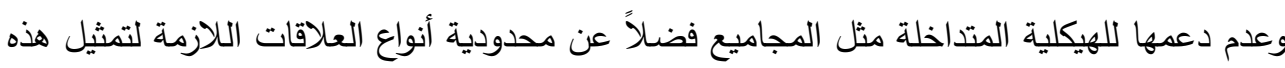

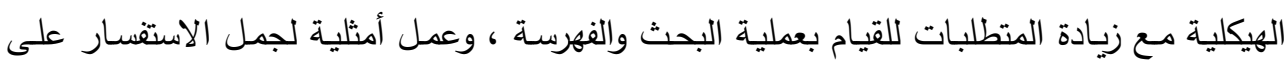

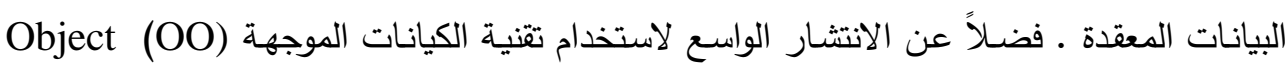
Oriented الموجهة(Object Oriented programming(OOP في قواعد البيانات وفرت خصـائص الكيانات والأصناف والوراثة والكبسلة والتجريد وتعدد الأشكال وإعادة الاستخدام [7][17] ـ ـ ولكن

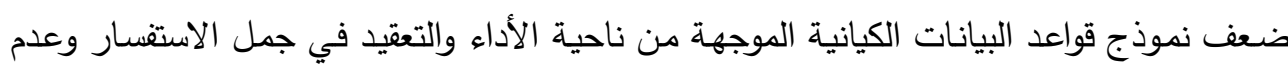

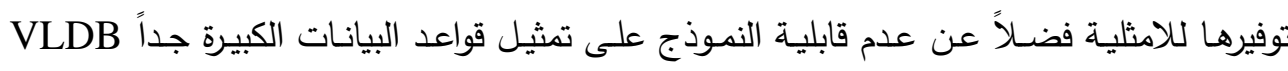


Very Large Data Base الكيانات العلائقية Object Relational Model الذي يقوم بتوسيع النموذج العلائقي من خلال ضم صفات نموذج قواعد البيانات الكيانية الموجهة إليه [9]. والثكل (1) يوضـح التطور الزمني لتوني لنظم إدارة قواعد البيانات [11].

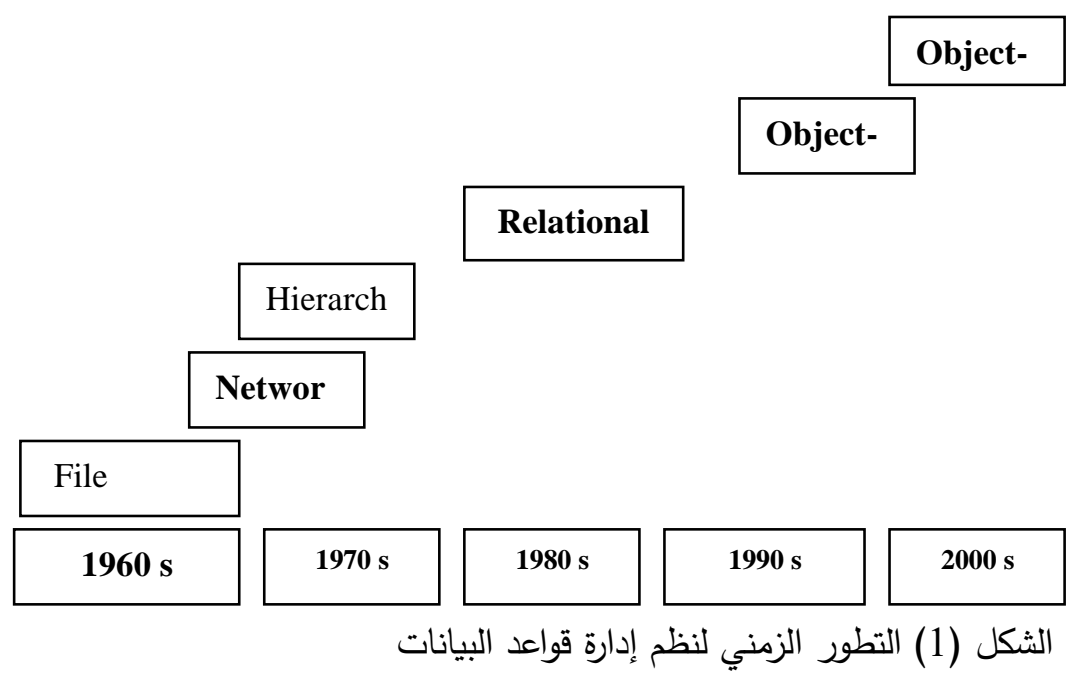

Object Type

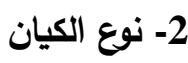

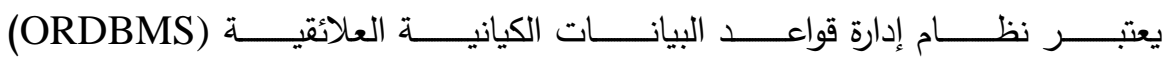

Object Relational Data Base Management System العلائقية Relational Data Base Management System (RDBMS) مضافاً إليه تقنية الكيانات الموجهة Oriented Object (OO) من خلال السماح للمستخدمين بتعريف نوع الكيان وهو نوع مركب يتم تكوينه بناءً على تعريف المستخدم ولا يكون معرفاً مسبقاً ويقوم

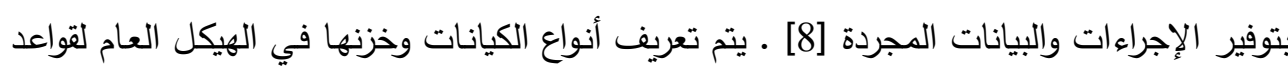

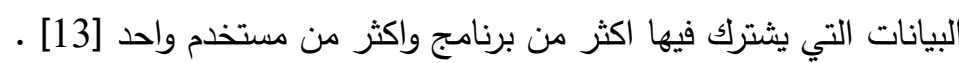

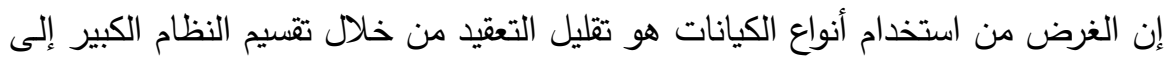

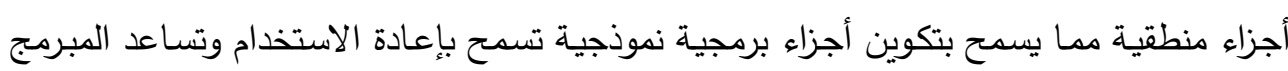

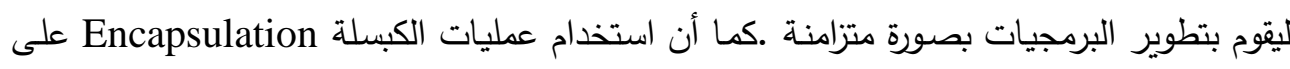

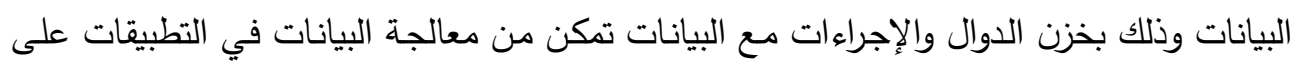

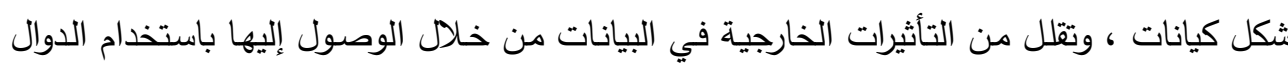


والإجراءات المرتبطة بها [8] وبذلك يمكن استخدام أنواع الكيانات لضم نموذج الكيانات مباشرةً مع

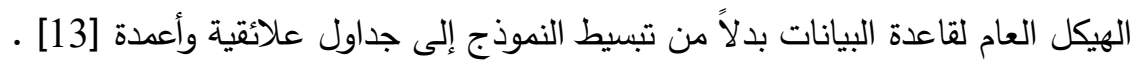

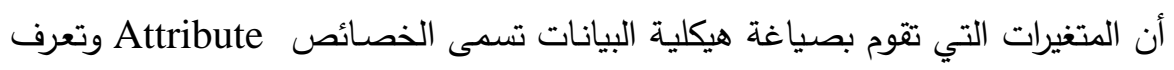

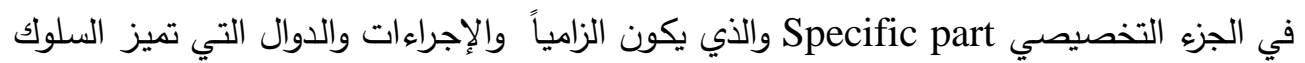
الخاص لنوع الكيان تسمى بالطرائق Methods وتعرف في جزء الجسم Body part الذي يكون اختيارياً . أخاصن إن النمذجة البيانيـة تعتمد على التحليل النصسي للمسألة وفيما يأتي وصف نصسي لنظام

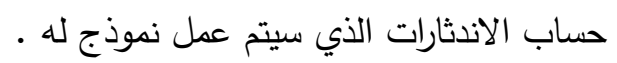

الاندثار (الاستهلاك) هو النقص التدريجي في قيمة الموجود الثابت لأسباب كثيرة منها

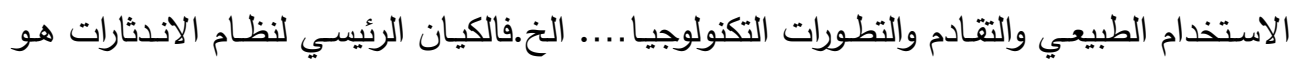

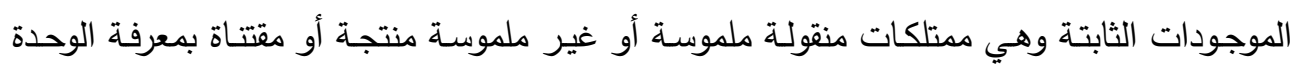

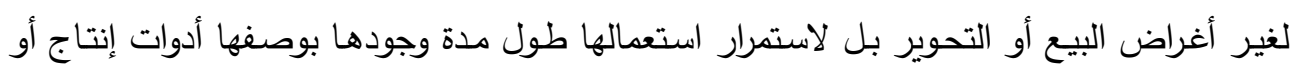

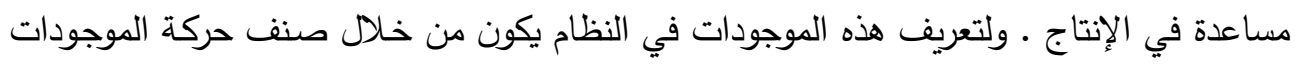

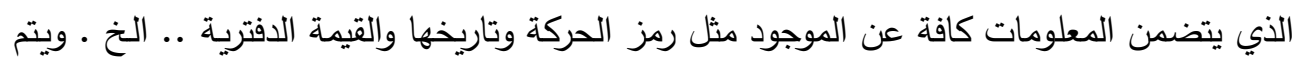

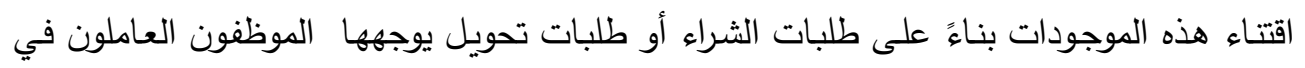

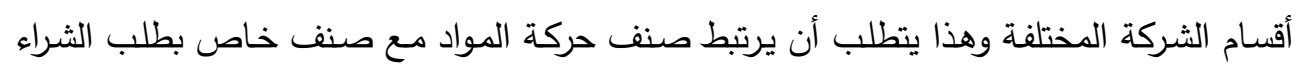
والذي يضم رقم الطلب وتاريخه وقائمة المواد المطلوبة وأسعارها والمبلغ الإجمالي إضافة إلى ارتباط

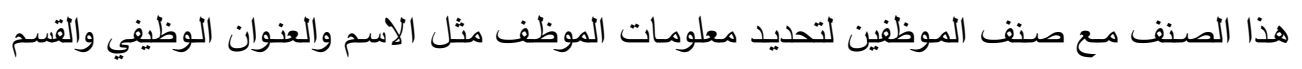
الذي يعمل فيه وتختلف الموجودات الثابتة من حيث طبيعتها واستخدامها وطريقة احتساب الاندثار السنوي عليها ، لذلك يتطلب أن يرتبط صنف حركة الموجودات مـ صنف الموجودات الأساسية

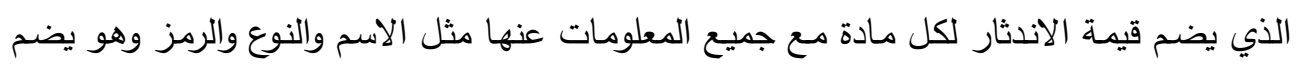
الموجودات الثابتة الملموسة (المادية) وهي التي يتم استخدامها في الوحدة الاقتصادية لغرض الإنتان الإنتاج

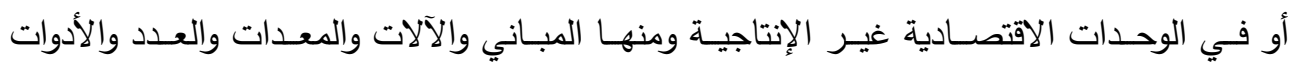

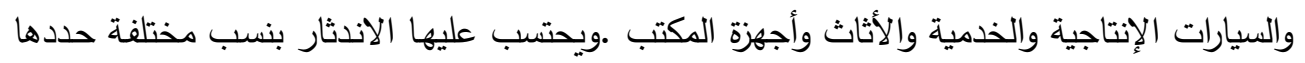

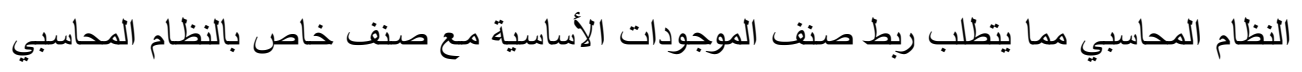

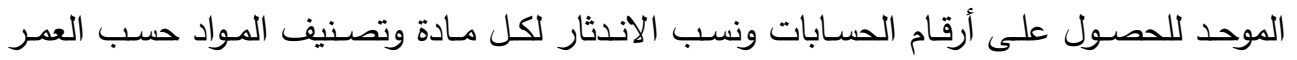
التقديري المتوقع لكل من هذه الموجودات . 
إن حساب الاندثارات هي طريقة محاسبية تهدف إلي توزيع تكلفة الموجودات الرأسمالية الملموسة أو قيمها الأساسية ناقصـاً قيمة الخردة (في حال وجودها) على الحيقة الحياة الإنتاجية المقدرة

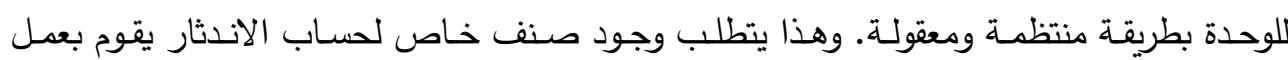

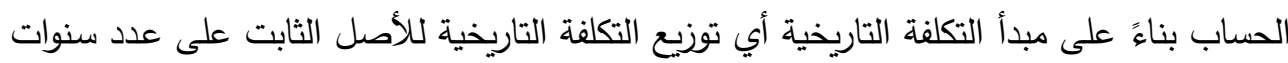

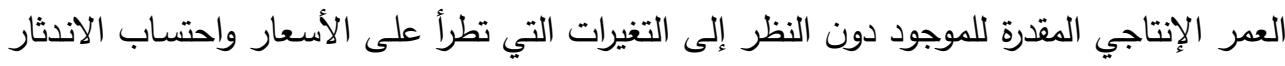
على هذه القيمة وعرضها بكشوفات ملحقة مع الحسابات الختامية لكي تتم مقارنتها والتعرف على على الإنى التغييرات المستمرة في قيمة الموجودات .فضلاً عن احتساب عمر الآلة منها عدد ساعات التشغيل

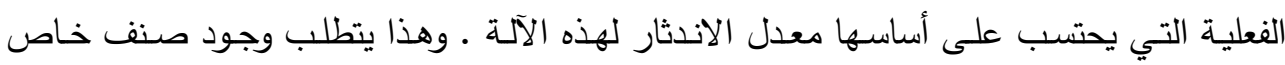

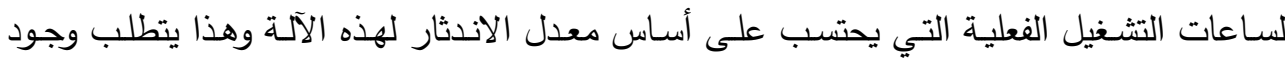

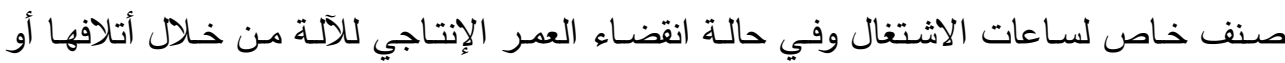

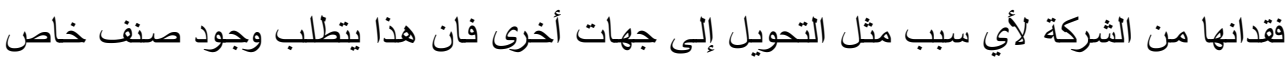
بحركة الثطب والتحويل للموجود وتتم هذه الحركة بإشراف لجان خاصـة تقوم بالعملية وهذا يتطلب

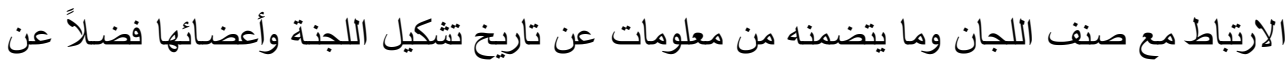

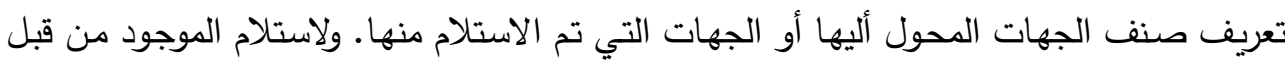

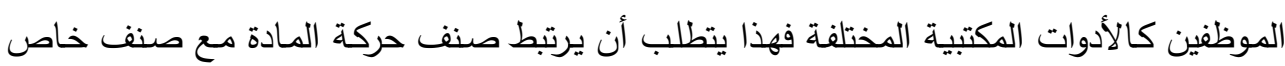
بالذمم لمعرفة الموظف المستخدم لهذا الموجود والقسم العامل فيه وهذا يتطلب عمل ربط بين هذه الأصناف باستخدام علاقات ذات تعدديات مختلفة.

والثـكل (2) يوضــح المخطط العـام لنظـام حسـاب الانـدثارات والمكتوب بلــغة النمـاذج

الموحدة UML) Unified Modeling Language) وتم فيه تعريف أنواع البيانات الخاصة extint_t و و نوع الكيان الخاص بالاندثارات

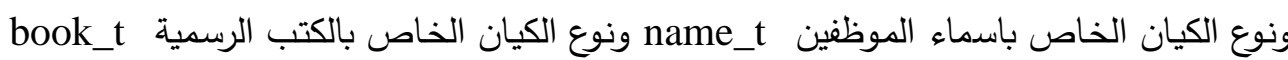

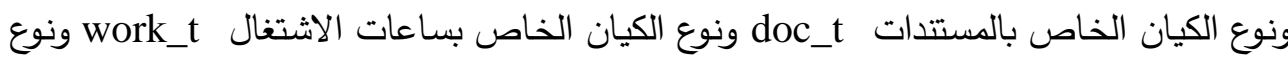

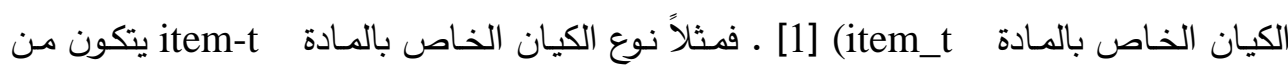

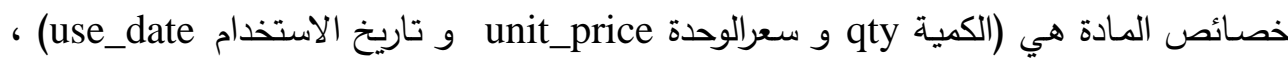

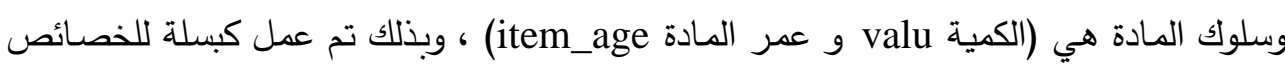
والطرائق في نوع الكيان [ 4] [ 8] [16]. والثكل(3) يوضسح المقطع البرمجي لتكوين نوع الكيان

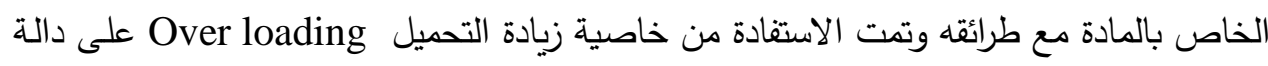




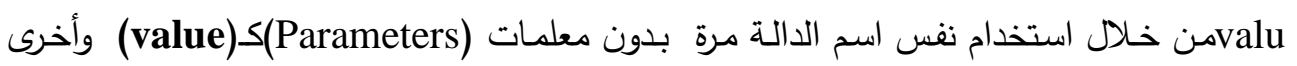

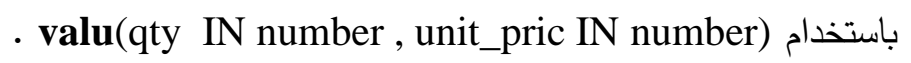

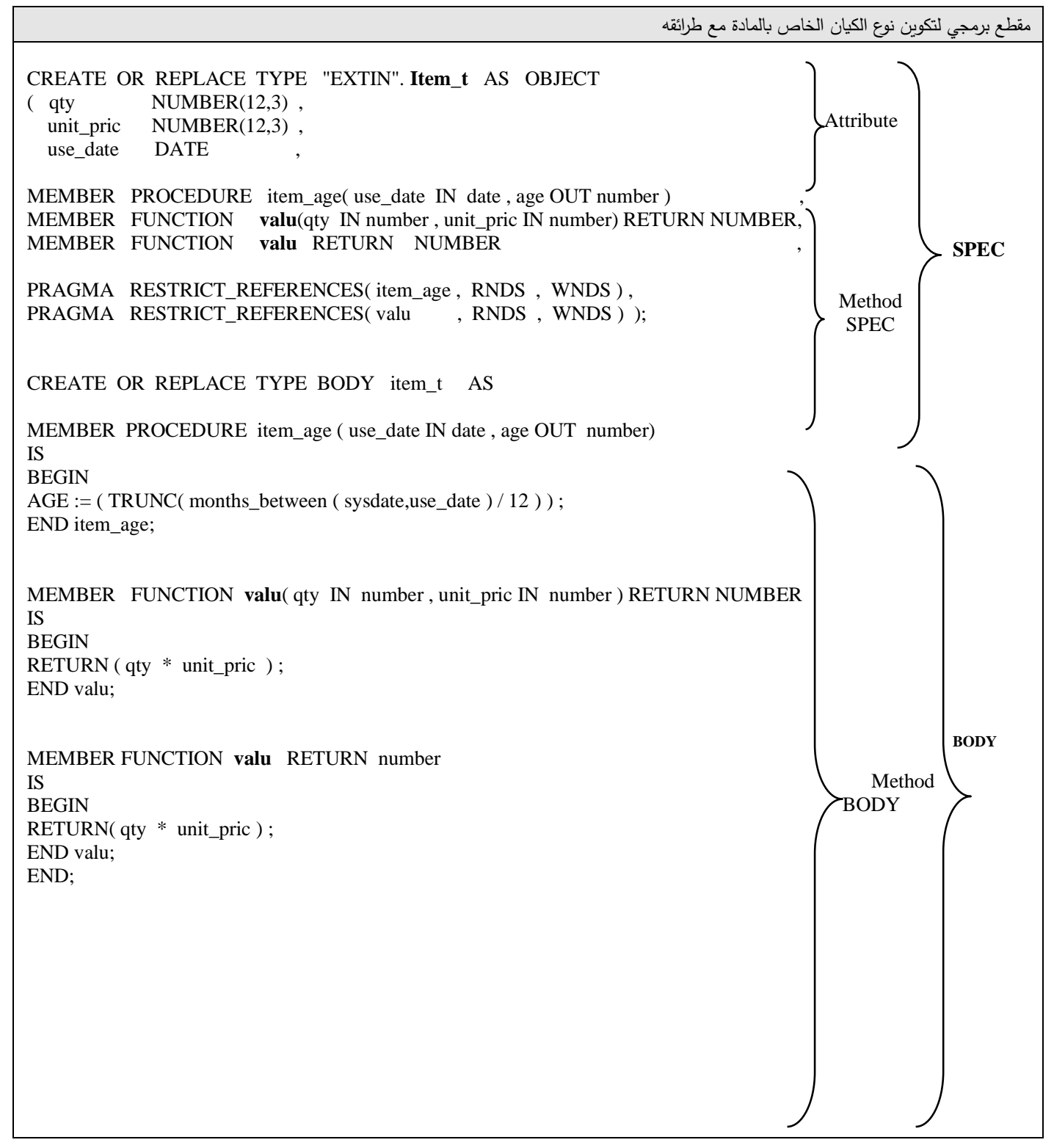

الشكل (3) نوع الكيان وطرائقه

Sharing Objects

3- المشاركة بين الكيانات 
تمتلك الكيانات معرفاً أحادياً يسمى بمعرف الكيان Object Identifier (ويختصر بـ

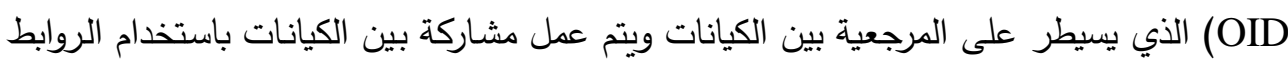

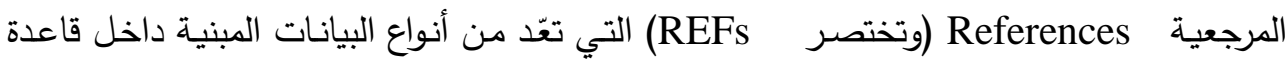
البيانات للغة المستخدمة (أو راكل) [8]. ومن فوائد استخدام REF هو تجنب الفهرسة والروابط المكلفة وعدم تكرار البيانات غير الضرورية وعند القيام بتحديث الكيانات المشتركة فان التغيير يحدث في مكان واحد فقط ويقوم المرجعية لعF

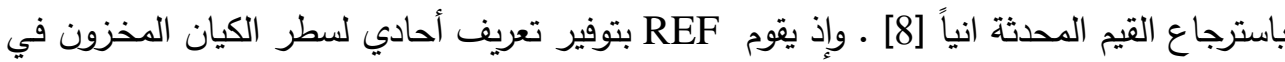

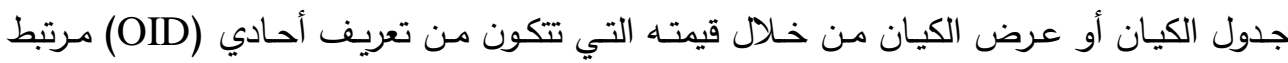
بجدول الكيانات ومعرف لسطر الكيان (ROWID) المخزون في الجدول وهذا يوفر وصولاً سريعاً إلى الكيان المطلوب ويسهل عملية الربط بين الكيانات [12] .وتعطي نموذجاً لمجموعة الروابط تجاه

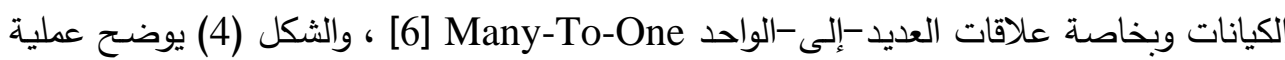

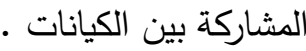
Globally unique

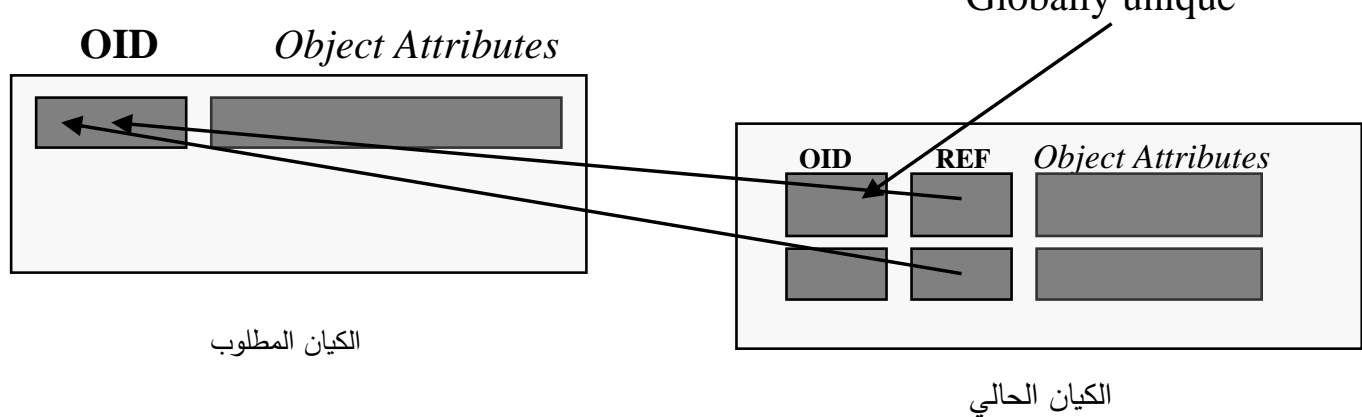
الثكل (4) المشاركة بين الكيانات

وفي نظام الاندثارات تم عمل مراجع بين نوع الكيان الخاص بالذمم Borou مع نوع الكيان الخاص

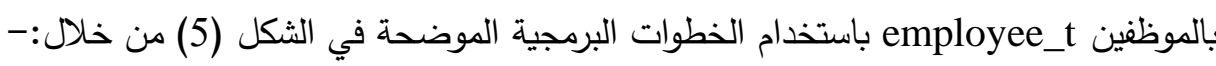

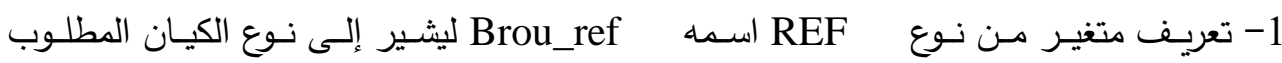
.employee_t 2- كتابة جملة Select لإرجاع قيمة OID الخاصة بالكيان المطلوب employee_t من عرض الكيان الخاص بالموظفين employee_t إلى المتغير المعرف Brou_ref. مقطع برمجي للحصول على المراجع في جدول الذمم 


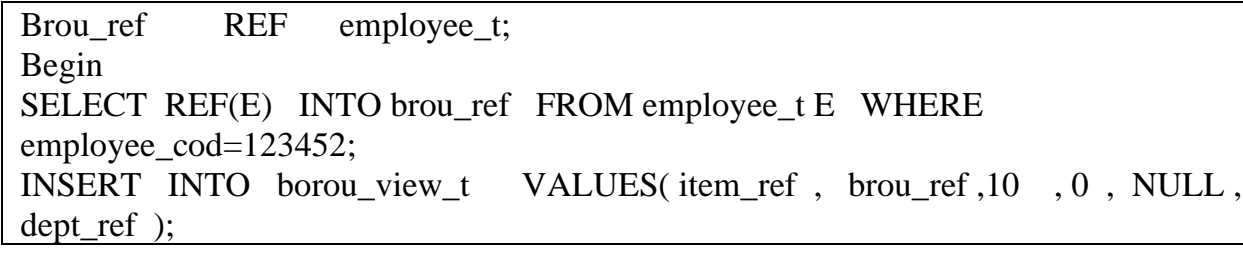

$$
\text { الثكل(5) الحصول على المراجع }
$$

أما عملية استرجاع البيانات المشار إليها باستخدام REF فتتم من خلال ما يسمى بإعادة

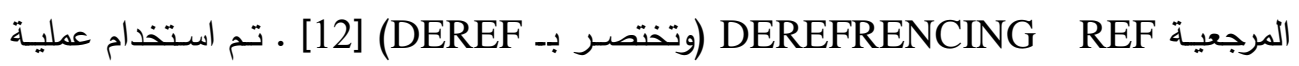
DEREF مع جملة Select لتقوم بإرجاع قيمة الكيان من الجدول المزدوج DUAL إلى متغير

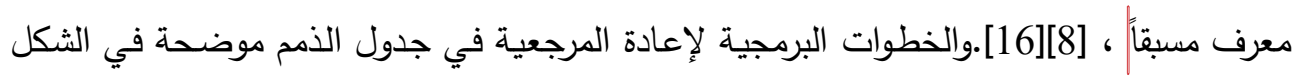

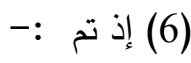

1- ليشريف متغير من نوع الكيان V_borou. ليشير الى نوع الكيان المطلوب femployee_t

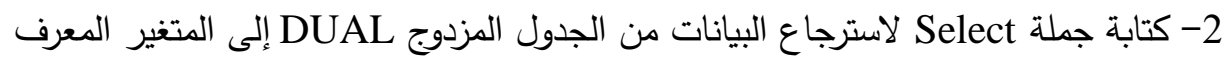
. DEREF باستخدام عملية V_borou مقطع برمجي لإعادة استخدام المراجع مع جدول الذمم

\section{DECLARE}

V_borou employee_t;

\section{BEGIN}

IF INSERTING THEN

SELECT DEREF (:NEW.brou_cod) INTO V_borou FROM DUAL; INSERT INTO borou (cod, conv_dat, conv_cod, brou_cod, qty , fback, note ,dept_cod )

VALUES ( V_item.cod , :NEW.conv_dat ，V_conv.employee_cod , V_borou.employee_cod :NEW.qty , :NEW.fback , :NEW.note , V_dept.dept_cod );

$$
\text { الثكل (6) إعادة المرجعية }
$$

\section{Collection Types}


هي مجموعة من الكيانات التي تتجمع في متغير واحد من نوع بيانات المجاميع (الجدول

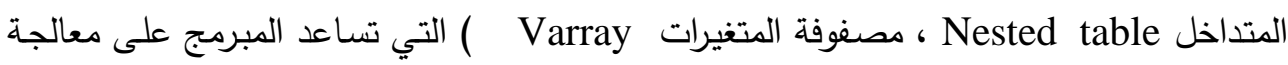

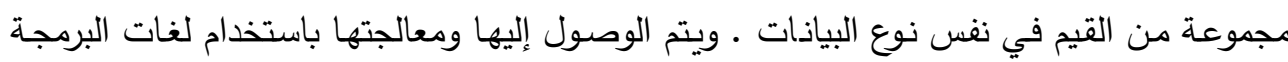

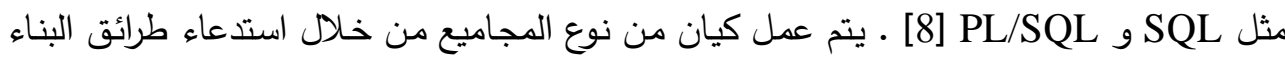

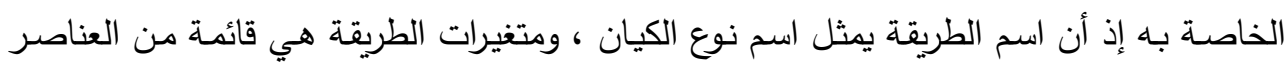

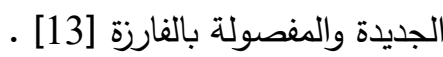

\section{Varying array}

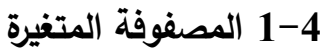

وهي مصفوفة ذات بعد واحد تحتوي على عناصر بيانات مرتبة من النوع القياسي أو من

نوع بيانات الكيان وكل عنصر في المصفوفة له تسلسل يعتمد على موقع العنصر في المصفوفة

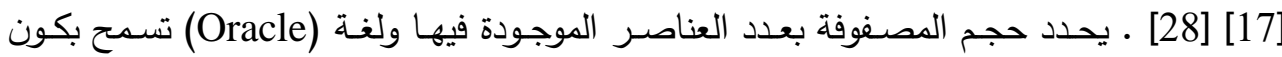

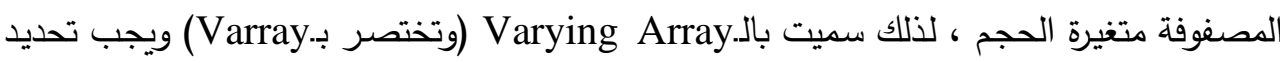
أعلى حجم للمصفوفة عند تعريف المستخدم لها [12] [13] .تعرف ال. Varray بوصفها عموداً في جدول البيانات ، وجميع البيانات الموجودة فيها يتم استرجاعها ومعالجتها في خطوة واحدة عند عملية استخراج البيانات وعلى النحو الموضح في الثكل (7) ـ لذلك تعّد

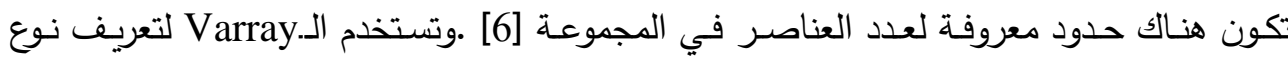

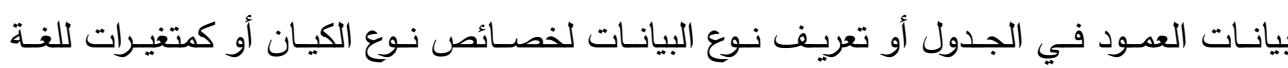

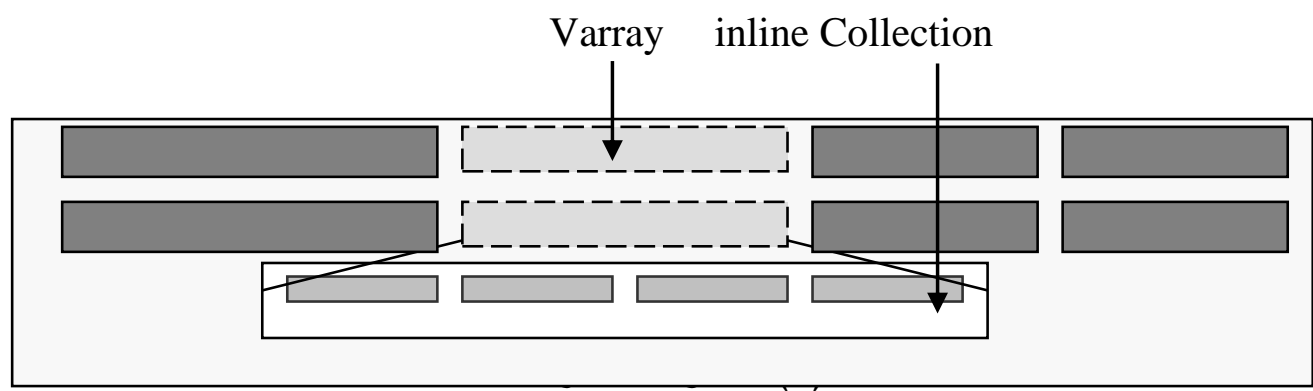


وقد تم تعريف Vاصـة بأرقام الهواتف والدرجات الوظيفية في نظام حساب Varray

الاندثارات والخطوات البرمجية اللازمة لتعريف مصفوفة المتغيرات Varray لارقام الهواتف موضحة

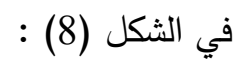

تكوين نوع بيانات الكيان الخاصة بارقام الهواتف من خلال إيعاز Create Type.

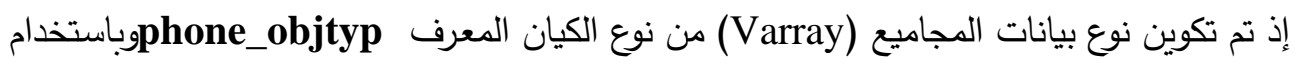
إيعاز Create Type تضمين نوع بيانات المجاميع Phone_list_t كعود في نوع الكيان Pagent_t

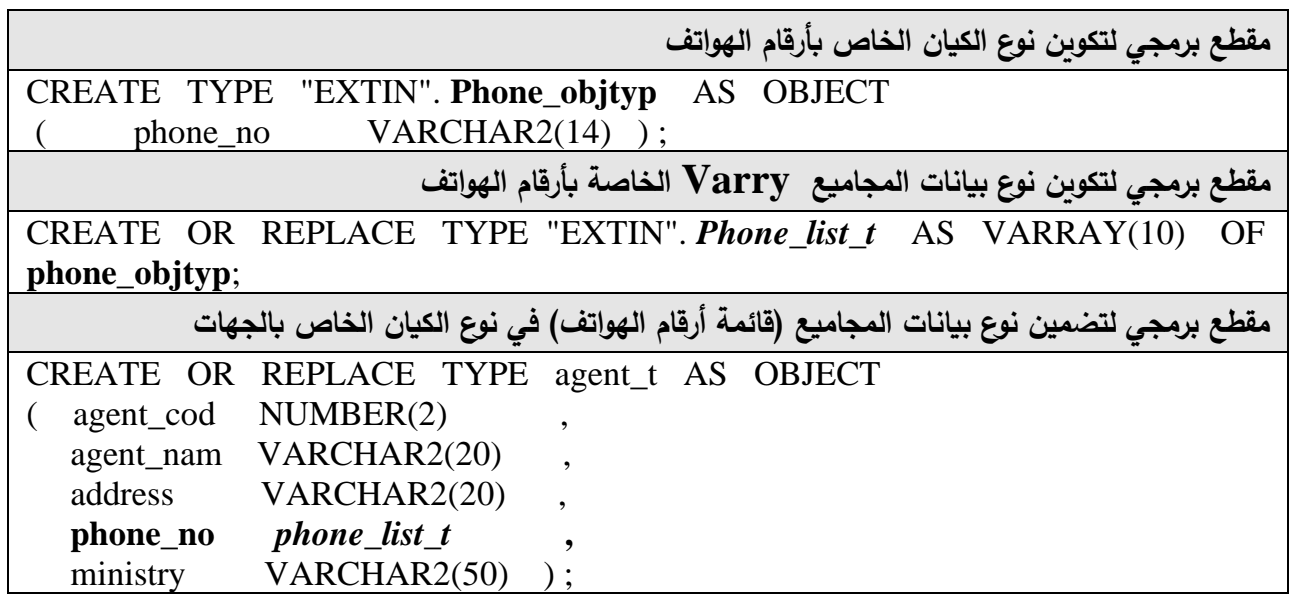

الثكل (8) تكوين مصفوفة المتغيرات

\section{Nested Table}

4-2 الجدول المتداخل

Outer هو جدول تتم إضافته إلى قاعدة البيانات بوصفه عموداً في الجدول الخارجي

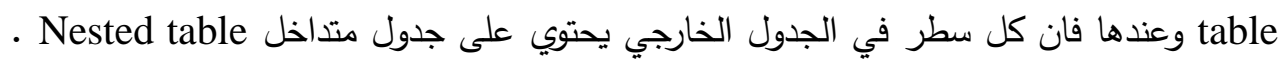
والعناصر الخاصة بالجدول المتداخل تكون غير مرتبة وهي من أنواع البيانات القياسية أو من أنواع لئل بيانات الكيان وتخزن هذه العناصر في جدول داخلي منفصل Inner table وبطريقة مشابهة لعلاقة

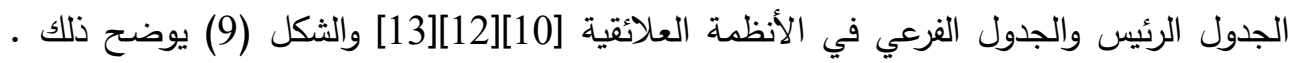

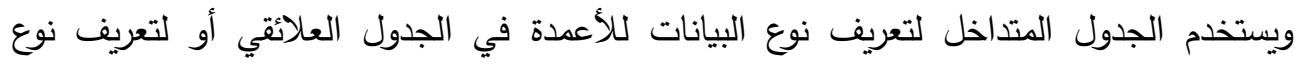
البيانات لخاصية من خصائص نوع الكيان أو كمتغيرات للغة PL/SQL . ويتم التعامل مع الجدول المتداخل بدلاً من مصفوفة المتغيرات عندما تكون البيانات غير محددة الحجم وترتيبها غير مهم أو أو عند عمل فهرسة أو استعلام لخاصية من خصائص الجدول المتداخل [2] . 


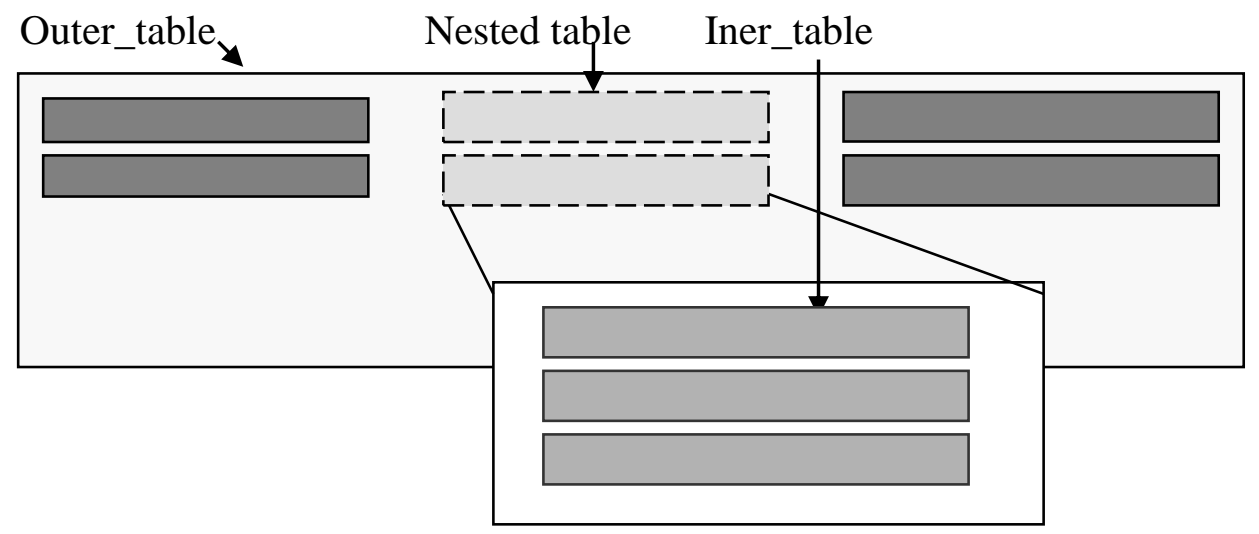

$$
\text { الثكل (9) الجدول المتداخل }
$$

والخطوات البرمجية اللازمة لتعريف الجدول المتداخل Nested table التي تم تطبيقها

في نظام الاندثارات على جدول المواد المطلوب شراؤها Items من خلال تعريفه S Nested : table

1-تكوين نوع الكيان item_typ الخاص بالجدول الداخلي الذي يعطي هيكلية للجدول المتداخل مقطع برمجي لتكوين نوع الكيان الخاص بالمواد المطلوبة

CREATE OR REPLACE TYPE "EXTIN". item_typ AS OBJECT

( order_id NUMBER(6),

qty NUMBER(12,3),

unit_pric NUMBER(12,3),

use_date DATE );

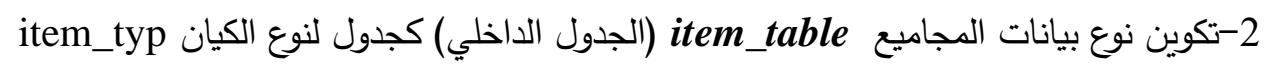

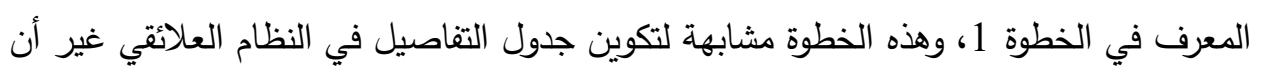
العمود الخاص بالمفتاح الأجنبي غير موجود.

مقطع برمجي لتكوين جدول الكيان الخاص بالمواد المطلوبة CREATE OR REPLACE TYPE "EXTIN".item_table AS TABLE OF Item_typ; 
3- تكوين جدول قاعدة البيانات salenest_table(الجدول الخارجي) وهذه الخطوة مشابهة لتكوين الجدول الرئيس في النظام العلائقي ، وتم استخدام جملة Nested table لتكوين الجدول المتداخل

$$
\text { من خلال دمج الجدول الداخلي بوصفه عموداً في الجدول الخارجي. }
$$

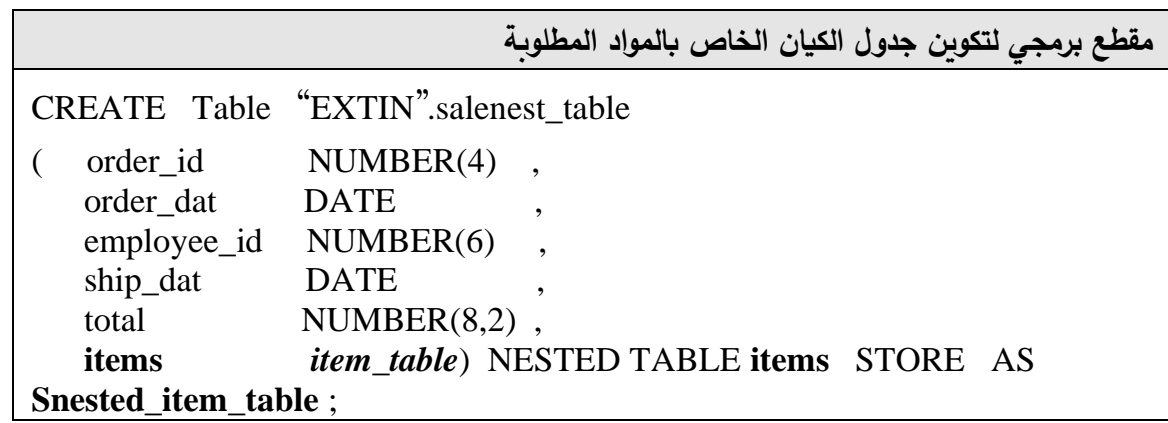

$$
\text { الشكل (10) تعريف الجدول المتداخل }
$$

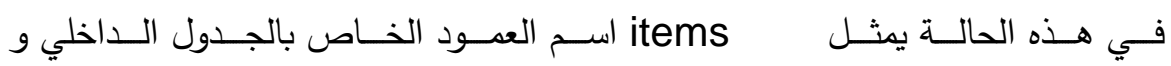

Snested_item_table خزنه في نفس مساحة الجداول Table Spaces الخاصة بالجدول الخارجي.

1-2-4 استخدام لغة معالجة البيانات في الجدول المتداخل

\section{Using Data Manipulating Language in Nested Table}

تمت معالجة البيانات الخاصة بالجدول المتداخل باستخدام لغة معالجة البيانات DML.

والثكل (11) يوضح العمليات التي تم تنفيذها على الجدول المتداخل الخاص بطلبات الثراء والمواد

$$
\text { المطلوبة والذي تم تكوينه في الخطوات البرمجية السابقة. }
$$


مقطع برمجي لعملية معالجة البيانات في الجدول المتداخل لطلبات الشراء DECLARE
Item_count NUMBER;
BEGIN

-- -

INSERT INTO salenest_table VALUES ( 1 , sysdate, null, sysdate, 100, item_table (item_typ $(1,10,20$, sysdate $)))$;

INSERT INTO salenest_table VALUES ( 2 , sysdate, null , sysdate, 2000 , s Item_table(item_typ $(2,100,20$,sysdate $))$ );

$$
\text { - - }
$$

INSERT INTO THE ( SELECT items FROM Snested_item_table WHERE order_id=2) VALUES ( ITEM_TYP $(1,543,10$, sysdate));

$$
\text { --عملية تحديث الجدول الخارجي-- }
$$

UPDATE salenest_table SET items = item_table(item_typ(9,30,99999,null))

$$
\text { WHERE order_id }=1 \text {; }
$$

$$
\text { - - عملية تحديث الجدول المتداخل-- }
$$

UPDATE THE ( SELECT items FROM Snested_item_table WHERE ORDER_id = 2) SET use_date $=$ sysdate WHERE order_id $=2$;

SELECT COUNT(*) INTO item_count

$$
\text { --عملية الاختيار من الجدول المتداخل--- }
$$

FROM THE ( SELECT items FROM Snested_item_table WHERE ORDER_id=1)

$$
\text { WHERE use_date }<=\text { sysdate; }
$$

DELETE FROM salenest_table WHERE order_id=1;

$$
\text { --عملية الحذف من الجدول المتداخل-- }
$$

DELETE THE ( SELECT items FROM Snested_item_table WHERE ORDER_id=2) WHERE USE_DATE < sysdate; END;

$$
\text { الثكل (11) لغة معالجة البيانات في الجدول المتداخل }
$$

تم الاعتماد على لغة النماذج لأنها توفر رموزاً سهلة الفهم والاستخدام في تمثيل نموذج قواعد البيانات الكيانية الذي مكن من الحصول على نموذج للتطبيق

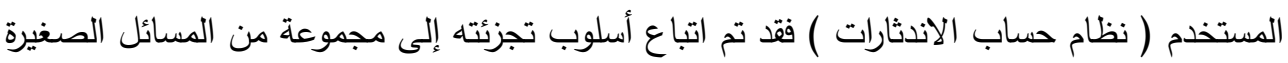
مثل الجزء الخاص بنظام الأفراد والجزء الخاص بنظام المشتريات والجزء الخاص بنظام الحسابات

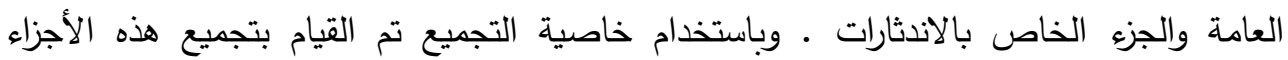
بسهولة لتكوين النموذج العام للنظام .وتم التعامل مع كل جزء من النماذج السابقة بوصفه نموذجاً

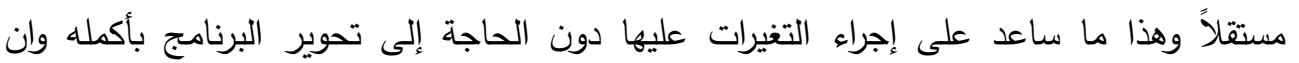


معالجة الأخطاء التي ظهرت في مرحلة البرمجة كانت محددة ضمن كل نموذج دون أن تؤثر في

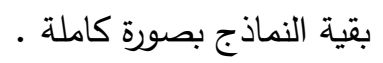

إن استخدام نظام إدارة قواعد البيانات الكيانية العلائقية وفر إمكانيات جديدة في إدارة

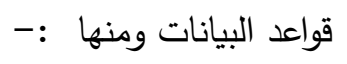

مكن استخدام أنواع البيانات الكيانية من إغناء النظام من خلال تجهيزه بأنواع يعرفها المستخدم (UDT) User Define Type الخاصة بكيانات إدارة الأعمال التي تعامل كالأنواع القياسية المبنية في النظام مثل CHAR,DATE مع توفير حماية ضد سوء الاستخدام لهذه الأنواع

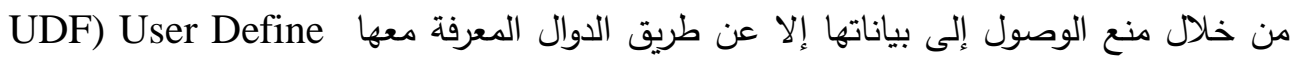

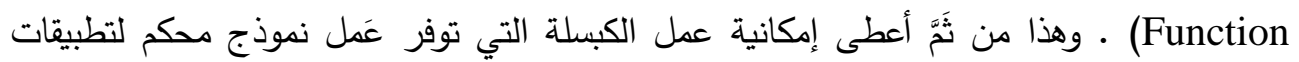
الكيانات.

إن استخدام مراجع الكيانات REFs مكن من الحصول على نموذج من مجاميع علاقات

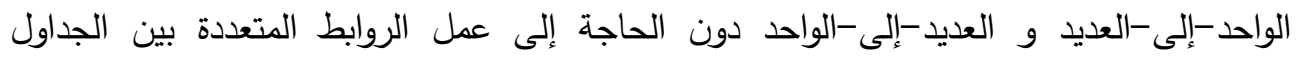

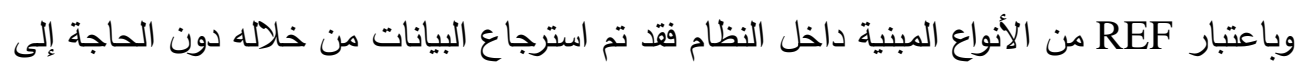
بناء جمل SQL بل تم ذلك من خلال عملية طلب واحدة. مكن استخدام أنواع بيانات المجاميع (Varray,Nested table) من تضمين البيانات

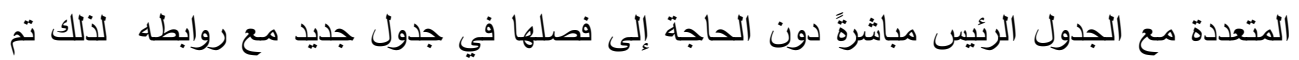

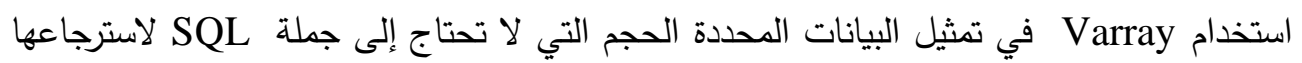
وهذا له فائدة في تسريع عملية استرجاع البيانات نفسها مقارنة بالجدول العلائقي والجداول المتداخلة 
المصادر

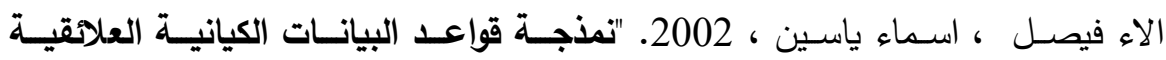

باستخدام اوراكل"، رسالة ماجستير /جامعة الموصل - كلية علوم الحاسبات والرياضيات

دايتر كارول ، 2000 ـ" اوراكل8 بايبل "، دار الفاروق للنشر والتوزيع / مركز التعريب

$$
\text { والترجمة / مصر ماروك }
$$

[3] Beaverton O. R, ".2001 .Object-Relational and ObjectOriented Database System “, http://www.software .ibm/is/swservers/database.

[4] Johnny O ; .Allan R. L ，."2000 ， Experiences from

"Object-Relational Programming in Oracle8 Cot/4-06-

V1.4, http://www.cit.dk/COT/reports/reports/case4/.4v1,./cot-4-06-v1.4.pdf.

[5] Beaverton O. R, .2001 .Object-Relational and ObjectOriented Database System", http://www.software .ibm/is/swservers/database.

[6] Lonsdale M, ."1999 . Is Performance a Reason for Using Oracle8 Object ?", http://www.softlab.co.uk new/ uploads /ISPERFO8. $p d f$

[7] Oracle-developer, "2000 . Introduction to Object-Relational Database Development", http://www.kingtraining.com/downloads /o8diffs-paper.pdf.

[8] Portfolio T., 1999. "PL/SQL Users Guide and Reference" http://www.technet.oracle.com

[9] Ramakanth $\mathrm{S} ;$. Konda $\mathrm{D}$, ".2001 .ObjectRelational Database System-The Road ahead http://www.acm.org/crossroads/xrd S7-3/ordbms.html 
[10] Oracle Technical White Paper, "2001. Simple Strategies for Complex Data : Oracle9i Object-Relational Technology" "http://www.ont . oracle. com /products/oracle9i/pdf/iot_twp.pdf. , "

[11] Rob P. ; Coronel C. , 2000 . Data base System Design , Implement and Management , $4^{\text {th }}$, course technology.

[12] Russell J , ."1999 . Application Developers Guide" ObjectRelational Features, http://www.thinkspark ·co.uk /ioug/PLSQL New Features in oracle8i.pdf.

[13] Sikora Y ; .Peter J.L , .2001 .Object to Object Communication , http//:www.odtug.com.

[14] Ratio Group Ltd, 2001 . "Persistence : Implementing Object Over a Relation Database version 1.0" , http://www.ratio.co.uk.

[15] Ulman J. D ; .Widom J , ."1997 • Afirst Course in Database System ", Prentic-Hall International, Inc.

[16] Whithead A. N . 2000 . Object-Relational Oracle8 and PL/SQL8 " ,http://www.polito.itivrea /inofrmazioni /passaggio/ dispenseinseif/ basi/PLSQL8.pdf

[17] Woodger Computing Inc, 2001. "Object Data Base", http://www.wci object DB capabilities.htm. 